\title{
Quantification of T-wave Morphological Variability Using Time-warping Methods
}

\author{
J. Ramírez ${ }^{1}$, M. Orini ${ }^{2}$, E. Pueyo ${ }^{1}$ and P. Laguna ${ }^{1}$ \\ ${ }^{1}$ Biomedical Signal Interpretation and Computational Simulation (BSICoS) group, Aragón Institute of Engineering Research (I3A), IIS \\ Aragón, University of Zaragoza, Zaragoza, Spain and Biomedical Research Networking Center (CIBER), Zaragoza, Spain. \\ 2 Institute of Cardiovascular Science, University College London, London, UK.
}

\begin{abstract}
The aim of this study is to quantify the variation of the T-wave morphology during a 24-hour electrocardiogram (ECG) recording. Two ECG-derived markers are presented to quantify T-wave morphological variability in the temporal, $d_{w}$, and amplitude, $d_{a}$, domains. Two additional markers, $d_{w}^{\mathrm{NL}}$ and $d_{a}^{\mathrm{NL}}$, that only capture the non-linear component of $d_{w}$ and $d_{a}$ are also proposed. The proposed markers are used to quantify $T$ wave time and amplitude variations in 500 24-hour ECG recordings from chronic heart failure patients. Additionally, two mean warped T-waves, used in the calculation of those markers, are proposed to compensate for the rate dependence of the $T$-wave morphology. Statistical analysis is used to evaluate the correlation between $d_{w}, d_{w}^{\mathrm{NL}}, d_{a}$ and $d_{a}^{\mathrm{NL}}$ and the maximum intra-subject RR range, $\Delta R R$. Results show that the mean warped T-wave is able to compensate for the morphological differences due to $R R$ dynamics. Moreover, the metrics $d_{w}$ and $d_{w}^{\mathrm{NL}}$ are correlated with $\Delta \mathbf{R R}$, but $d_{a}$ and $d_{a}^{\mathrm{NL}}$ are not. The proposed $d_{w}$ and $d_{w}^{\mathrm{NL}}$ quantify variations in the temporal domain of the $T$-wave that are correlated with the RR range and, thus, could possibly reflect the variations of dispersion of repolarization due to changes in heart rate.
\end{abstract}

Keywords - Electrocardiogram, morphological variability, repolarization, T-wave, time-warping.

\section{INTRODUCTION}

The T-wave reflects the spatio-temporal dispersion of repolarization of the ventricular myocytes [1]. Thus, if ionic exchanges during ventricular repolarization or propagation of the electrical impulse throughout the ventricles suffer from any abnormalities, this will be reflected on the morphology of the T-wave [2]. Steep slopes of the T-peak-to-end (Tpe) dynamics, considered as a non-invasive marker to some extent related to enhanced spatio-temporal dispersion of repolarization restitution, have been suggested to be linked to the generation of ventricular arrhythmias that could lead to sudden cardiac death, while flat slopes indicate mechanical heart fatigue predisposing to [3]. The hypothesis of this study is that the information contained in the morphology of the T-wave may provide stronger risk prediction markers than those obtained when using the Tpe interval only. Overall shifts in the temporal domain, or misalignments between T-waves, might complicate the comparison and corrupt the measurement of Twave morphological variability. Linear and non-linear temporal re-parameterization (warping) techniques have been used to overcome this limitation, align electrocardiogram (ECG) waves and measure amplitude differences with improved accuracy [4]. However, the warping information has never been used as a marker to assess the T-wave variability in the temporal domain.

The main objective of the study is to assess the relationship between the maximum RR range and four ECGderived markers, $d_{w}$ and $d_{a}$, quantifying T-wave morphological variability in the temporal and amplitude domains, respectively, and their non-linearly restricted versions, $d_{w}^{\mathrm{NL}}$ and $d_{a}^{\mathrm{NL}}$. Such relationship is evaluated in 24-hour Holter ECGs from chronic heart failure (CHF) patients.

\section{MethodS}

\section{A. Mathematical Framework}

Let's consider two T-waves, $\boldsymbol{f}^{r}\left(\boldsymbol{t}^{r}\right)=$ $\left[f^{r}\left(t^{r}(1)\right), \ldots, f^{r}\left(t^{r}\left(N_{r}\right)\right)\right]^{\top} \quad$ and $\quad f^{s}\left(\boldsymbol{t}^{s}\right)=$ $\left[f^{s}\left(t^{s}(1)\right), \ldots, f^{s}\left(t^{s}\left(N_{s}\right)\right)\right]^{\top}$, where $\boldsymbol{t}^{r}=\left[t^{r}(1), \ldots, t^{r}\left(N_{r}\right)\right]^{\top}$ and $\boldsymbol{t}^{s}=\left[t^{s}(1), \ldots, t^{s}\left(N_{s}\right)\right]^{\top}$ and $N_{r}$ and $N_{s}$ being the total duration of $\boldsymbol{t}^{r}$ and $\boldsymbol{t}^{s}$, respectively, as illustrated in Figure 1(a). We take $\boldsymbol{f}^{r}\left(\boldsymbol{t}^{r}\right)$ as the reference T-wave and $\boldsymbol{f}^{s}\left(\boldsymbol{t}^{s}\right)$ as the T-wave to be compared with respect to $\boldsymbol{f}^{r}\left(\boldsymbol{t}^{r}\right)$.

Let $\gamma\left(\boldsymbol{t}^{r}\right)$ be the warping function that relates $\boldsymbol{t}^{r}$ and $\boldsymbol{t}^{s}$, such that the composition $\left[\boldsymbol{f}^{s} \circ \gamma\right]\left(\boldsymbol{t}^{r}\right)=\mathbf{f}^{s}\left(\gamma\left(\boldsymbol{t}^{r}\right)\right)$ denotes the re-parameterization or time domain warping of $\boldsymbol{f}^{s}\left(\boldsymbol{t}^{s}\right)$ using $\gamma\left(\boldsymbol{t}^{r}\right)$. The square-root slope function (SRSF) was proposed [5] to find the optimal warping function in a well-defined geometrical space by warping the SRSFs of the original Twaves, defined as the square-root of the derivative of $f(t)$, considering the sign:

$$
\boldsymbol{q}_{f}(\boldsymbol{t})=\operatorname{sign}(\dot{\boldsymbol{f}}(\boldsymbol{t})) \sqrt{|\dot{\boldsymbol{f}}(\boldsymbol{t})|}
$$

The SRSF of $\boldsymbol{f}^{r}\left(\boldsymbol{t}^{r}\right)$ and $\boldsymbol{f}^{s}\left(\boldsymbol{t}^{s}\right), \boldsymbol{q}_{f^{r}}\left(\boldsymbol{t}^{r}\right)$ and $\boldsymbol{q}_{f^{s}}\left(\boldsymbol{t}^{s}\right)$, respectively, are shown in Figure 1 (b). The optimal warp- 

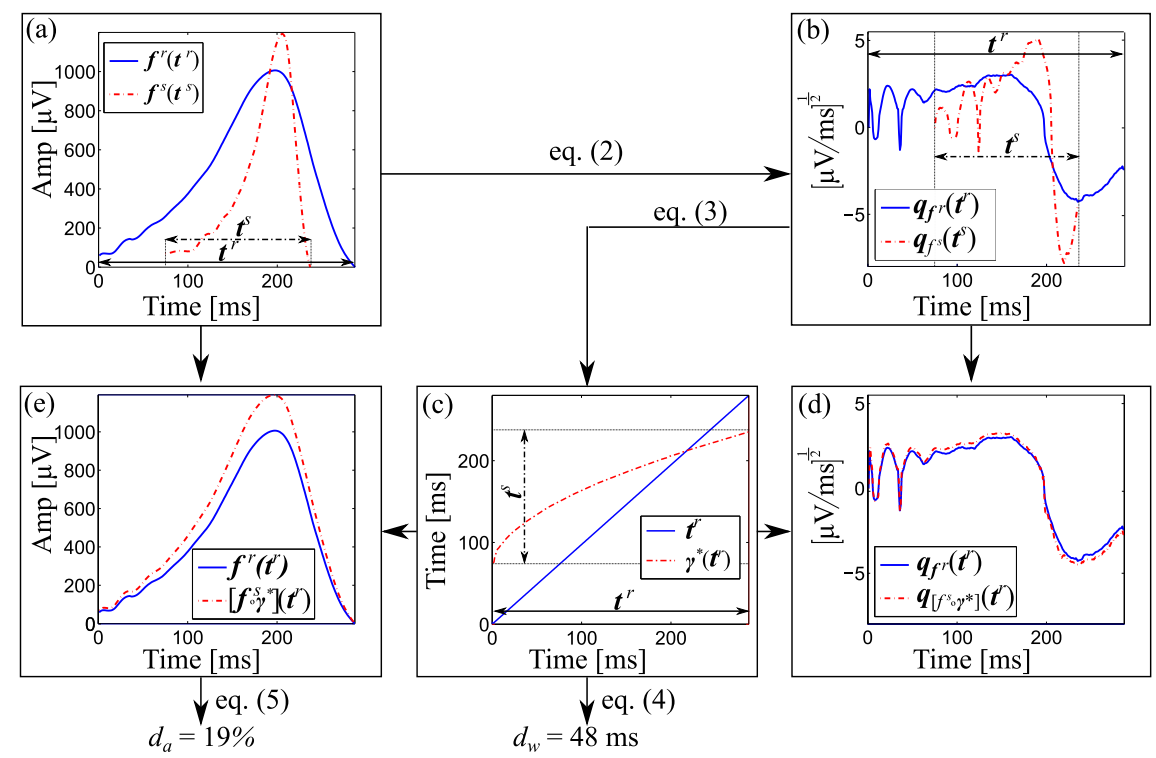

Fig. 1: Diagram flow illustrating the computation of $d_{w}$ and $d_{a}$. (a) Reference T-wave (solid blue) and a T-wave presenting both time and amplitude variability (shorter duration and larger amplitude) (dashed red). (b) Applying eq. (1) we obtain their respective square-root slope functions. (c) Optimizing eq. (2) with the "Dynamic Programming" algorithm, we get $\boldsymbol{\gamma}^{*}\left(\boldsymbol{t}^{r}\right)$, the warping function that optimally relates $\boldsymbol{t}^{r}$ and $\boldsymbol{t}^{s}$. (e) The re-parameterization of $\boldsymbol{f}^{s}\left(\boldsymbol{t}^{s}\right)$ using $\boldsymbol{\gamma}^{*}\left(\boldsymbol{t}^{r}\right)$ leads to $\left[\boldsymbol{f}^{s} \circ \boldsymbol{\gamma}^{*}\right]\left(\boldsymbol{t}^{r}\right)$, the warped T-wave with no remaining time domain variability, and only presenting amplitude variability. (d) Square-root slope functions of the reference (solid blue) and warped (dashed red) T-waves.

ing function is the one that minimizes the amplitude difference between the SRSF of $\boldsymbol{f}^{r}\left(\boldsymbol{t}^{r}\right)$ and $\boldsymbol{f}^{S}\left(\gamma\left(\boldsymbol{t}^{r}\right)\right), \boldsymbol{q}_{f^{r}}\left(\boldsymbol{t}^{r}\right)$ and $\boldsymbol{q}_{\left[f^{s} \circ \gamma\right]}\left(\boldsymbol{t}^{r}\right)=\boldsymbol{q}_{f^{s}}\left(\gamma\left(\boldsymbol{t}^{r}\right)\right) \sqrt{\dot{\gamma}\left(\boldsymbol{t}^{r}\right)}$, respectively [5]:

$$
\begin{aligned}
\gamma^{*}\left(\boldsymbol{t}^{r}\right) & =\underset{\gamma\left(\boldsymbol{t}^{r}\right)}{\arg \min }\left(\left\|\boldsymbol{q}_{f^{r}}\left(\boldsymbol{t}^{r}\right)-\boldsymbol{q}_{\left[f^{s} \circ \gamma\right]}\left(\boldsymbol{t}^{r}\right)\right\|\right) \\
& =\underset{\gamma\left(\boldsymbol{t}^{r}\right)}{\arg \min }\left(\left\|\boldsymbol{q}_{f^{r}}\left(\boldsymbol{t}^{r}\right)-\boldsymbol{q}_{f^{s}}\left(\gamma\left(\boldsymbol{t}^{r}\right)\right) \sqrt{\dot{\gamma}\left(\boldsymbol{t}^{r}\right)}\right\|\right) .
\end{aligned}
$$

The dynamic programming algorithm was used to obtain the solution of this optimization problem [6]. The optimal warping function, $\gamma^{*}\left(\boldsymbol{t}^{r}\right)$, that optimally warps $\boldsymbol{f}^{r}\left(\boldsymbol{t}^{r}\right)$ and $\boldsymbol{f}^{s}\left(\boldsymbol{t}^{s}\right)$ is shown in Figure 1 (c). The warped T-wave, $\boldsymbol{f}^{s}\left(\gamma^{*}\left(\boldsymbol{t}^{r}\right)\right)$ is shown in Figure 1 (e), together with the reference T-wave, $\boldsymbol{f}^{r}\left(\boldsymbol{t}^{r}\right)$, while their corresponding SRSFs are shown in Figure 1 (d).

A metric, $d_{w}$, was defined that quantifies the level of warping needed to optimally align any two T-waves as the average of the absolute difference value between $\boldsymbol{\gamma}^{*}\left(\boldsymbol{t}^{r}\right)$ and $\boldsymbol{t}^{r}$ :

$$
d_{w}=\frac{1}{N_{r}} \sum_{n=1}^{N_{r}}\left|\gamma^{*}\left(t^{r}(n)\right)-t^{r}(n)\right|,
$$

The amplitude difference between $\boldsymbol{f}^{r}\left(\boldsymbol{t}^{r}\right)$ and $\boldsymbol{f}^{s}\left(\boldsymbol{\gamma}^{*}\left(\boldsymbol{t}^{r}\right)\right)$ is quantified as the area contained between $f^{r}\left(t^{r}\right)$ and $\boldsymbol{f}^{s}\left(\boldsymbol{\gamma}^{*}\left(\boldsymbol{t}^{r}\right)\right)$, normalized by the L2-norm of $\boldsymbol{f}^{r}\left(\boldsymbol{t}^{r}\right)$ :

$$
d_{a}=\frac{e_{a}}{\left\|e_{a}\right\|} \cdot \frac{\left\|\boldsymbol{f}^{s}\left(\boldsymbol{\gamma}^{*}\left(\boldsymbol{t}^{r}\right)\right)-\boldsymbol{f}^{r}\left(\boldsymbol{t}^{r}\right)\right\|}{\left\|\boldsymbol{f}^{r}\left(\boldsymbol{t}^{r}\right)\right\|} \times 100,
$$

where $\frac{e_{a}}{\left\|e_{a}\right\|}$, with $e_{a}=\sum_{n=1}^{N_{r}}\left(f^{s}\left(\gamma^{*}\left(t^{r}\right)\right)-f^{r}\left(t^{r}\right)\right)$, accounts for the sign.

By fitting $\gamma^{*}\left(\boldsymbol{t}^{r}\right)$ with a linear regression, $\gamma_{1}^{*}\left(\boldsymbol{t}^{r}\right)$, and measuring the mean deviation of $\gamma^{*}\left(\boldsymbol{t}^{r}\right)$ with respect to this regression, a quantification of the level of non-linear warping can be obtained:

$$
d_{w}^{\mathrm{NL}}=\frac{1}{N_{r}} \sum_{n=1}^{N_{r}}\left|\gamma^{*}\left(t^{r}(n)\right)-\gamma_{l}^{*}\left(t^{r}(n)\right)\right|,
$$

where $\gamma_{l}^{*}\left(\boldsymbol{t}^{r}\right)$ is the linear fitting of $\boldsymbol{\gamma}^{*}\left(\boldsymbol{t}^{r}\right)$.

Regarding $d_{a}$, by normalizing the warped T-waves, nonlinear amplitude differences not due to linear scaling can be quantified, which might possibly be caused by heterogeneous dispersion of repolarization times:

$$
d_{a}^{\mathrm{NL}}=\left\|\frac{\boldsymbol{f}^{r}\left(\boldsymbol{t}^{r}\right)}{\left\|\boldsymbol{f}^{r}\left(\boldsymbol{t}^{r}\right)\right\|}-\frac{\boldsymbol{f}^{s}\left(\boldsymbol{\gamma}^{*}\left(\boldsymbol{t}^{r}\right)\right)}{\left\|\boldsymbol{f}^{s}\left(\boldsymbol{\gamma}^{*}\left(\boldsymbol{t}^{r}\right)\right)\right\|}\right\| \times 100 .
$$

\section{B. Study population}

Consecutive patients with symptomatic chronic heart failure (CHF) corresponding to New York Heart Association classes II and III were enrolled in the MUSIC (MUerte Súbita 
en Insuficiencia Cardiaca) study, a prospective, multicenter study designed to assess risk predictors for cardiovascular mortality in ambulatory patients with CHF [7]. The 24-hour Holter recordings of 500 patients with sinus rhythm were available for the present study. Each recording consisted of three orthogonal ECG leads, sampled at $200 \mathrm{~Hz}$.

\section{Signal Preprocessing and T-wave extraction}

Preprocessing of the ECG signals included low-pass filtering at $40 \mathrm{~Hz}$ to remove electric and muscle noise, cubic splines interpolation for baseline wander removal and ectopic beats detection. Principal component analysis was calculated lead-wise to emphasize the T-wave components, improve its delineation and enhance morphological differences. The first principal component was delineated using a single-lead technique [8], and the T-waves were confined from the T-wave onset and T-wave end delineation marks.

Before applying the warping algorithm, the reference and the studied T-waves were aligned according to their gravity centers, so that $\gamma^{*}\left(t^{r}\right)$ is only dependent on changes in the T-wave morphology, and not on global shifts.

\section{Calculation of T-wave morphology variability}

First, the histogram of the RR series was calculated during the entire 24-h recording, and it was divided into bins of $10 \mathrm{~ms}$ wide. Then, only the bins having at least 50 values were selected (bins above the horizontal dotted line in Figure 2). Next, two RR bins, distributed symmetrically around the median RR (Figure 2, green arrow), were chosen as those defining the maximum intra-subject $R R$ range, $\Delta R R$, for the study (Figure 2, orange bins). Then, the T-waves corresponding to the beats associated with the RR intervals within these two bins were considered for the analysis.

The, the mean warped T-waves of those T-waves selected from each RR bin were calculated [9]. These mean warped Twaves are representatives of the average T-wave morphology at each corresponding RR interval value.

Finally, the morphological differences between both mean warped T-waves were quantified using $d_{w}, d_{w}^{\mathrm{NL}}, d_{a}$ and $d_{a}^{\mathrm{NL}}$. The separation of $\gamma^{*}\left(\boldsymbol{t}^{r}\right)$ from $\boldsymbol{t}^{r}$, quantified by $d_{w}$ and $d_{w}^{\mathrm{NL}}$ [9], measures the morphological differences in the time domain between the two mean warped T-waves. Note that if this line corresponded to the diagonal, no temporal transformation would be needed meaning that the morphological differences would be non-existent. The amplitude difference between these warped T-waves would be quantified by $d_{a}$ and $d_{a}^{\mathrm{NL}}$.

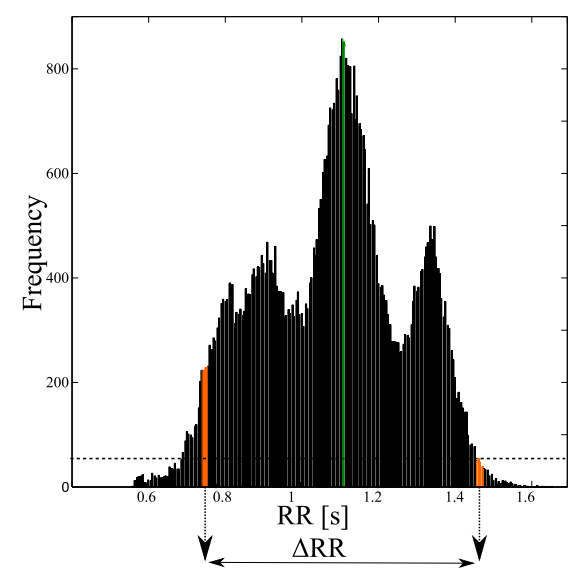

Fig. 2: RR histogram with bins of $R R=10 \mathrm{~ms}$. Green bin shows the median $\mathrm{RR}$ interval value. Orange bins indicate the RR values defining the maximum intra-subject range.

\section{RESULTS AND DISCUSSION}

The morphology of the T-wave is highly dependent on the history of previous RR intervals [10]. As seen in Figure 3, panel (b.1), the morphology of the T-wave coming from a history of previous RR interval values longer than the current one (panel a.1) is different from that in panel (b.3), which corresponds to a T-wave coming from a history of previous RR interval values shorter than the current one (panel b.1). Due to the fact that the mean warped T-wave is not just a simple signal averaging, but it rather stretches (warps) the temporal domains of the T-waves [9], prior to regular averaging, it is able to compensate for the morphological differences due to the different histories of RR interval values (c.1).

The median (interquartile range) value of $d_{w}$ was 16.4 (11.3) ms. These values were 6.1 (4.5) $\mathrm{ms}$ for $d_{w}^{\mathrm{NL}},-22.6$ (45.5) $\%$ for $d_{a}$ and 8.0 (10.7) \% for $d_{a}^{\mathrm{NL}}$. The median (interquartile range) value of the maximum intra-subject $R R$ range was $0.43(0.21) \mathrm{s}$. The Spearman correlation coefficients between $\Delta \mathrm{RR}$ and $d_{w}, d_{w}^{\mathrm{NL}}, d_{a}$ and $d_{a}^{\mathrm{NL}}$ were $0.50(<0.001), 0.37$ $(<0.001), 0$ (0.956 and $0.06(0.153)$, respectively. This suggests that the morphological variations captured by $d_{w}$ and $d_{w}^{\mathrm{NL}}$ are correlated with the variations in the heart rate as it is expected from the fact that the temporal domain of the Twave varies with heart rate. Future studies might consider the restitution of such variations in the temporal domain of the T-wave as a marker related to the slope of dispersion of repolarization restitution and elucidate to what extent the heart rate corrected $d_{w}$, estimating the slope of the T-wave morphology restitution, could have potential for arrhythmic risk prediction. 


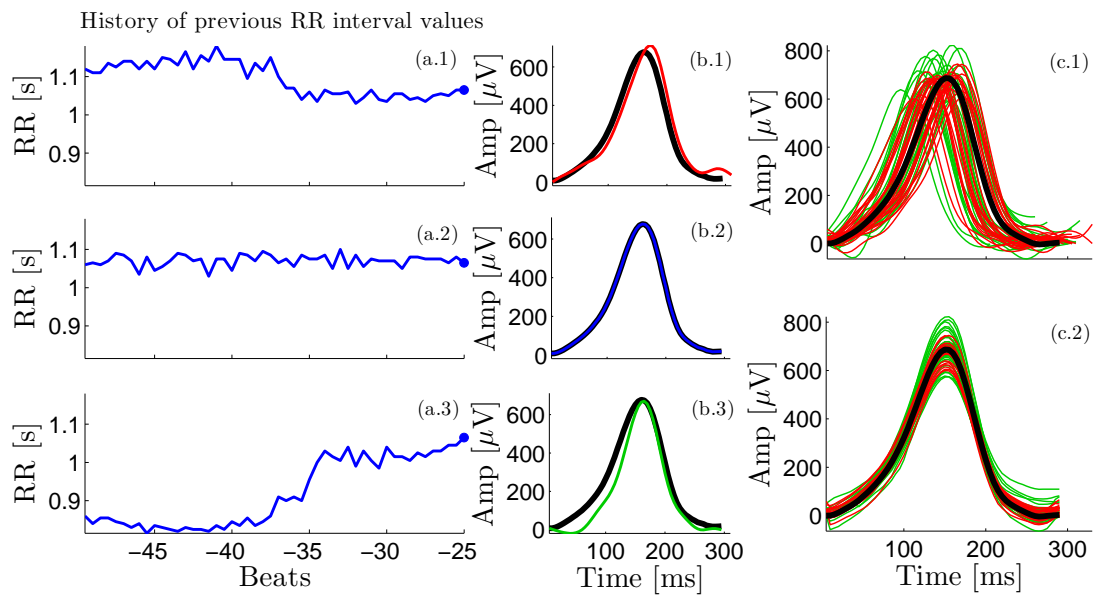

Fig. 3: Compensation for the T-wave morphology rate dependence. Panels (a.1-3) show three different dynamics of RR. Panels (b.1-3) show the mean warped T-wave (black) and the T-wave morphology of the current beat. Red, blue and green colors indicate that the previous RR interval values were longer, similar and shorter, respectively, than the current one. Panel (c.1) shows the 50 T-waves from a particular RR bin. Panel (c.2) shows the 50 T-waves warped with respect to the mean warped T-wave.

\section{CONCLUSION}

In this study, a mean warped T-wave was proposed to compensate for the rate-dependency of the T-wave morphology in 24-hour Holter ECG recordings. This mean warped T-wave may be clinically useful as a representative manifestation of ventricular repolarization dispersion at a certain $R R$ value. The metrics quantifying variations in the temporal domain of the T-wave, $d_{w}$ and $d_{w}^{\mathrm{NL}}$, which use the proposed mean warped T-wave in their calculation, were shown to be correlated with the maximum intra-subject RR range. Thus, $d_{w}$ and $d_{w}^{\mathrm{NL}}$, could help quantifying the variability in the dispersion of ventricular repolarization, and might have potential for arrhythmic risk stratification. Future studies will also assess the arrhythmic risk predictive value of the restitution of the T-wave morphology, quantified by normalizing $d_{w}$ and $d_{w}^{\mathrm{NL}}$ by $\Delta \mathrm{RR}$, since we postulate it could act as a surrogate of the dispersion of repolarization restitution slope.

\section{CONFLICT OF INTEREST}

The authors declare that they have no conflict of interest.

\section{ACKNOWLEDGEMENTS}

This work was supported in part by projects TIN2013-41998$\mathrm{R}$ and DPI2016-75458-R from Spanish Ministry of Economy and Competitiveness (MINECO), Spain, MULTITOOLS2HEART from CIBER-BBN through Instituto de Salud Carlos III, Spain and by Aragón Government, Spain and from European Social Fund (EU) through BSICoS group. The computation was performed at the High Performance computing platform of the NANBIOSIS ICTS, CIBER-BBN and I3A, Zaragoza, Spain.

\section{REFERENCES}

1. V. M. F. Meijborg et. al. . Electrocardiographic T-wave and its relation with ventricular repolarization along major anatomical axes Circ: Arrhythmia Electrophysiol. 2014;7:524-531.

2. K. Gima and Y. Rudy . Ionic current basis of electrocardiographic waveforms: a model study Circ Res. 2002;90:889-896.

3. J. Ramírez et. al. . Automatic SVM classification of sudden cardiac death and pump failure death from autonomic and repolarization ECG markers. J Electrocardiol. 2015;48:551-557.

4. M. Schmidt et. al. . Two-dimensional warping for onedimensional signals: conceptual framework and application to ECG processing IEEE Trans Signal Process. 2014;62:55775588.

5. J. D. Tucker et. al. . Generative models for functional data using phase and amplitude separation Comput Stat Data Anal. 2013;61:50-66.

6. D. P. Bertsekas . Dynamic programming and optimal controlch. 1. Belmont, MA 1995.

7. R. Vázquez et. al. . The MUSIC Risk score: a simple method for predicting mortality in ambulatory patients with chronic heart failure. Eur Heart J. 2009;30:1088-1096.

8. J. P. Martínez et. al. . A wavelet-based ECG delineator: evaluation on standard databases IEEE Transactions on Biomedical Engineering. 2004;51:570-581.

9. J. Ramírez et. al. . Variability of ventricular repolarization dispersion quantified by time-warping the morphology of the T-waves. IEEE Trans Biomed Eng. 2016;DOI:10.1109/TBME.2016.2614899.

10. E. Pueyo et. al. . Characterization of QT interval adaptation to $\mathrm{RR}$ interval changes and its use as a risk-stratifier of arrhythmic mortality in amiodarone-treated survivors of acute myocardial infarction IEEE Trans on Biomed Eng. 2004;51:1511-1520. 